Economía, Sociedad y Territorio, vol. viII, núm. 28, 2008, 1059-1066

\title{
Entre dilemas y oportunidades: las organizaciones comunitarias en la gestión local mexicana
}

\section{Between dilemmas and opportunities: Community organisations in the Mexican local management}

\author{
Arellano Gault, David (2006), Los Dilemas de LA GeSTión local Y \\ LAS ORGANIZACIONES COMUNITARIAS EN MÉxICO, CIDE, MÉXICO, 268 PP. \\ ISBN 968-7420-39-1
}

En el contexto local mexicano, caracterizado por sus procesos de cambio y complejidad, estudiar las relaciones entre organizaciones comunitarias (OCs) y las organizaciones gubernamentales nos permite analizar la dinámica de las interacciones y las consecuencias que se derivan de estas relaciones. En Los dilemas de la gestión local, David Arellano desarrolla una investigación que analiza los procesos de acercamiento, ajustes, regulación y cooperación entre organizaciones comunitarias y organizaciones burocráticas gubernamentales a fin de resolver problemas que requieren establecer reglas formales y procesos predeterminados para activar la acción pública. Las ocs son organizaciones caracterizadas por su flexibilidad y ambigüedad, lo cual les permite realizar tareas básicas en sus comunidades que no requieren institucionalizar sus actividades. El problema surge cuando estas organizaciones llevan a cabo tareas complejas que las obligan a establecer conexiones con las organizaciones gubernamentales. Arellano plantea que en estos procesos de intercambio muchas ocs sucumben y otras logran resistir a la exigencia burocrática de contar con reglas, procesos, objetivos y metas más formales, esto es, en la necesidad de institucionalizar la relación. ¿Qué sucede cuando estas ocs interactúan con la burocracia? ¿Cuáles son los principales elementos de esta interacción?

Para explicar estos procesos de intercambio entre ocs y gobierno, el autor analiza, vía los estudios de caso, cuatro municipios mexicanos ubicados en la región central: Zaragoza, Veracruz; Cuquío, Jalisco; Tierra Blanca, Guanajuato y Vanegas, San Luis Potosí. Estos municipios fueron elegidos a través de una minuciosa selección de variables que pudiesen hacerlos compa- 
rables y donde se encontrara evidencia de la participación entre ocs y gobierno. Todos corresponden a municipios rurales, enclavados en zonas geográficas de alta marginación y caracterizados por conflictos sociales o por control político; los casos se analizaron en la década de los noventa.

El trabajo se estructura en cuatro partes. El primer capítulo discute el análisis de las organizaciones centrado en la burocracia. Arellano inicia con el estudio de la burocracia desde el tipo ideal creado por Weber para explicar su potencial interpretativo de la realidad. El tipo ideal es una forma pura de la sociedad, y cada vez que la realidad se aleja del ideal es cuando podemos interpretar las fallas de la realidad más no explicarlas. La burocracia, desde la perspectiva del tipo ideal de Weber, es la forma más eficiente de orden y organización en una sociedad, ya que las reglas son impersonales, los puestos se asignan por mérito y los objetivos y metas son relativamente claros para los actores. Weber recalca la posición del poder en las relaciones entre la burocracia y el resto de los actores, señalando que la fórmula para explicar el poder es la probabilidad de los individuos para obedecer reglas. Arellano argumenta que el problema de la burocracia como tipo ideal no tomó en cuenta que los burócratas podrían buscar sus propios objetivos. De ahí que se incluya a los críticos de Weber: Robert K. Merton, Alvin Gouldner y P. Selznic que explican el dilema de la burocracia como una construcción social y como fuente de irregularidades donde objetivos y metas se pueden ver desplazados por las reglas. De igual forma, se toma en cuenta las relaciones de poder y el contexto en el que se desenvuelven los individuos como una fuente que determina las relaciones. Más adelante, Arellano incluye la visión ecológica de las organizaciones (Stewart R. Clegg) y la visión de la burocracia como mal necesario y desde la lógica del cálculo de los individuos dentro de las organizaciones (Michel Crozier) que establece que éstas son espacios donde los individuos utilizan su capacidad de cálculo y racionalidad para solucionar problemas. Este primer capítulo estructura las principales ideas sobre cómo interactúan y regulan su participación los individuos dentro de las organizaciones.

El segundo capítulo discute el enfoque metodológico utilizado en el estudio. Principia argumentando las dificultades y limitantes que tienen los métodos de investigación en particular cuando se trata del método de casos. El argumento central se orienta a explicar que en los estudios de caso se critica su alcance unitario y no 
holista y la tendencia de ciertos métodos a inclinarse por la parte cuantitativa y no cualitativa, con lo que existen problemas serios de interpretación o sesgo. Ello se ejemplifica al momento en que las preguntas codificadas siempre tienen origen en la posición del investigador. Estos problemas resultan porque en la parte cuantitativa las variables se pueden correlacionar, pero la correlación es distinta a la causalidad, elemento que sí se observa desde la parte cualitativa. En este sentido, la investigación extensiva privilegia los grandes espacios cuantitativos mientras los estudios intensivos se orientan a la parte cualitativa. Por otro lado, Arellano explica que el éxito de una investigación tiene que estar en función del establecimiento de los objetivos que ésta se plantea. A propósito de los estudios de caso, el autor propone: "En la investigación de un estudio de caso, el primer aspecto importante que debe considerarse es la definición del objetivo básico: el análisis de causalidades complejas con miras a proponer una interpretación plausible del fenómeno. La intencionalidad de los estudios de caso es el entendimiento de la causalidad en una realidad particular, o dentro de un marco específico, sin pretender generalizar. La meta no es predecir, sino entender" (pp. 71-72).

El tercer capítulo se enfoca a describir los estudios de caso. Inicia con la experiencia de la organización Comité de Defensa del Pueblo de Zaragoza (CDPZ), en Veracruz, que surge a partir de la crisis del municipio en la década de los setenta, cuando el gobierno federal prioriza la protección de zonas petroleras, marginando el desarrollo de la comunidad. En un principio, el CDPZ se posiciona como una organización antisistema que poco a poco va interactuando con la llegada de sacerdotes jóvenes con ideas cimentadas en la teología de la liberación, que a su vez generan una nueva plataforma de participación en la comunidad. El primer cambio se dio a través de la creación de las comunidades eclesiales de base (CEBs) que articulan la participación ciudadana y fortalecen al CDPZ. De este modo, dicho comité, apoyado por el Partido de la Revolución Democrática (PRD) gana la presidencia municipal de Zaragoza, con lo que inicia un proceso para constituir un gobierno ciudadano. En un principio, los problemas para sostener la participación se sortean vía los procesos de aprendizaje de la propia comunidad y a través de los comités vecinales, pero también por la propia supervivencia política de la CDPZ en la presidencia municipal. Arellano comenta que la experiencia de Zaragoza es interesante porque a pesar de que es una comunidad pobre, la capacidad de organización de la oc ha sido importante, aunque con el paso 
del tiempo ésta tenderá a desplazar la participación comunitaria por las reglas y la tecnología que necesariamente requieren otro tipo de relación entre gobierno y ocs.

El segundo caso es el municipio de Cuquío, Jalisco, caracterizado porque se encuentra en una zona política difícil y controlada por grupos priistas arraigados. En Cuquío se crean elementos para integrar una nueva estructura que es la de Accedde, la cual funciona como agencia de asesoría local, que aunado a la labor de las comunidades eclesiales de base constituyen a la Organización Campesina Independiente de Jalisco (OCIJ), vinculada al PRD. Esta organización gana las elecciones municipales en los años noventa a pesar de su dinámica ambigua, lo cual les genera problemas para establecer prácticas de gobierno y participación ciudadana, pero apostando en todo momento a la transparencia de procesos y dinámicas de gobierno. Al momento de llegar al poder municipal, los miembros de la OCIJ, junto con gente del PRD y de otros partidos políticos, logran llegar a acuerdos importantes, estableciendo como base de acción la participación ciudadana. Uno de los primeros resultados fue la creación del Consejo de Desarrollo Municipal de Cuquío (Codemuc), establecido como organismo independiente del gobierno municipal, encargado de determinar cuáles son las obras que debe construir el municipio. El éxito del Codemuc es grande pues muchas de las obras planteadas por ellos se tomaron en cuenta y se ha sustentado en dinámicas de transparencia y de rendición de cuentas y de organizar la organización para poder tener éxito. Sin embargo, el problema de la Codemuc es que aún se encuentra en la ambigüedad de sus reglas y normas de existencia y de trabajo. El hecho de que sea producto de la organización comunitaria pero apoyada por un partido político, plantea el problema de que muchos actores no se hayan podido incorporar y existe el temor de que la coyuntura política (la posibilidad de perder la presidencia municipal) termine con el proyecto. Por otro lado, la planeación, si bien exitosa, tampoco garantiza la viabilidad económica del ayuntamiento, por lo que se ha hecho necesario establecer algunas nuevas tendencias de ordenamiento y de institucionalización de este organismo.

El tercer caso es el municipio de Tierra Blanca, Guanajuato, el cual representa una particular manera de establecer relaciones entre las ocs y el gobierno en los ámbitos municipal y estatal. El caso inicia con la necesidad del gobierno estatal de negociar constantemente con formas autoritarias en el municipio, concretamente con la historia caciquil de Tierra Blanca, para poder 
implementar formas relativamente representativas de participación democrática que permitan generar beneficios a todos los habitantes. Arellano remarca la coyuntura política que sufrió el estado de Guanajuato en los años noventa cuando Vicente Fox perdió la elección de gobernador y el Partido Acción Nacional (PAN), a través de movilizaciones, bloqueó al ganador. El entonces presidente Carlos Salinas logró negociar con el PAN para colocar a Carlos Medina Plascencia como gobernador interino por tres años, representando ello una oportunidad para los panistas. Los Comités para la Planeación del Desarrollo Municipal (Copladem) de Tierra Blanca eran controlados por caciques y/o priistas. A la llegada del gobierno panista, con los apoyos del Programa Nacional de Solidaridad (Pronasol) y con las nuevas estructuras de gobierno, se intenta que en Tierra Blanca la conformación de los comités de planeación fuera relativamente democrática. El gobierno participa a través de la Coordinación del Desarrollo Regional (Codereg) para asesorar al Copladem municipal pero siempre manteniendo el respeto para ellos. El Copladem lo controlan los caciques y sus objetivos siempre se hacen a través de la negociación que se lleva a cabo entre los niveles de gobierno. La paradoja en Tierra Blanca es que a pesar de que mantienen una OC controlada por formas autoritarias, formalmente es eficiente y ello le garantiza el flujo de recursos en el ámbito estatal. El éxito depende de los procesos de negociación y los acuerdos más o menos establecidos entre los actores.

El último caso es el municipio de Vanegas, San Luis Potosí, enclavado en la zona desértica y con una larga historia de pobreza y marginación. En muchas ocasiones, su pobreza extrema les impide dimensionar nuevas estructuras de organización y formas de cooperación y liderazgos alternos. En este caso destaca la forma en la que una organización de gobierno, el DIF (Desarrollo Integral de la Familia) estatal, genera procesos de participación ciudadana en comunidades en extrema pobreza. Vanegas representó un reto para el Programa Alimentario de la Zona del Altiplano (PAZA) y el Programa Alimentario Escolar en la Zona Indígena (PAEZI) del gobierno del estado, encargados de vigilar y apoyar la alimentación infantil en zonas rurales. A través del PAZA el DIF estatal comenzó a implantar nuevas formas de cooperación para la nutrición de los niños, incentivando inicialmente entre los habitantes la autoparticipación, lo cual fue un éxito parcial pero suficiente para permitir que esta comunidad viera nacer un comedor infantil gestionado por el DIF y los habitantes. Estos pro- 
cesos se hicieron creando patrones de confianza entre los miembros de la comunidad. Sin embargo, al ser una participación ciudadana escasa, se comenzó a utilizar la amenaza por parte del DIF para incentivar una mayor participación ciudadana que también se logró aunque de forma más restringida. El PAZA logró que la comunidad solventara el tema del comedor para niños, observando que era factible generar una actividad económica sustentable a través de crear una granja de pollos propuesta por el DIF. El reto estaba entre las dos formas de organización. La primera era la comunitaria que no sabía cómo planear a largo plazo y la segunda era que el propio DIF no podía generar respuestas concretas ante las nuevas demandas. Lo que hace particular este proyecto es la propia ambición de generar primero participación ciudadana y después una participación empresarial en comunidades realmente marginadas. El problema lo enfrenta la transición de los espacios comunitarios a los espacios empresariales.

El cuarto capítulo comprende el análisis de resultados. Aquí se plantean algunas limitantes y criterios para acercarse a los estudios de caso. Arellano argumenta que la decisión de realizar estudios de caso se sustentó en tres elementos importantes: 1) el escaso control del investigador sobre la conducta y la situación real de las personas, lo que hace difícil restringir variables, 2) que el proceso de inducción es muy sensible a la complejidad histórica y social y 3) que el objetivo no puede ser generalizar o probar hipótesis. "Los estudios de caso sirven para comprender, con la mejor respuesta disponible, y para generar nuevas hipótesis y posibilidades teóricas" (p. 196). La comparación cruzada de los estudios de caso lleva al autor a explicar diferentes niveles de variables, algunas relativamente controlables y otras no. Estas últimas determinan ciertos patrones de explicación de la actividad de interacción entre las ocs y la burocracia.

Entre las conclusiones más interesantes destaca al patrón de comportamiento o etapas que tienen las ocs en su intercambio con la burocracia: 1) el compromiso, 2) el logro y 3) la autonomía económica. En estas tres etapas se observa una capacidad diferente de las ocs y de la burocracia para solucionar conflictos dependiendo de si la iniciativa es exógena (impuesta por la burocracia) o endógena (producida por la comunidad), de forma que los mecanismos de interacción y de solución de conflictos varían de una a otra. Otro elemento clave se relaciona con los problemas para mantener la acción colectiva cuando las metas y objetivos se vuelven más técnicos. Ello quiere decir que muchos logros 
entre los actores dependen de la capacidad que tienen para resolver los problemas.

Arellano explica que su trabajo propone dos contribuciones generales y una particular a la administración pública: la primera es reforzar la idea de que la burocracia es capaz de adaptarse y ser flexible, pero intentando al mismo tiempo reducir la incertidumbre haciendo uso de reglas impersonales o de autoridad. La segunda es la búsqueda de una nueva forma de organización flexible y sustentada en valores y acuerdos mutuos y compartidos por sus integrantes. La contribución particular es haber descubierto el proceso evolutivo en el que las ocs se relacionan exitosamente con las organizaciones burocráticas en la resolución de problemas.

Arellano cierra su reflexión explicando las potenciales ventajas de futuras investigaciones. El punto clave, argumenta, es entender que las ocs son organizaciones complejas que se construyen desde complejos contextos históricos, sociales y políticos. Además es un error pensar que son instrumentos cuando fluyen en espacios complejos. La búsqueda de la nueva organización se encuentra en una etapa preliminar, requiriendo a la vez de mucha inteligencia y tolerancia entre organizaciones totalmente distintas para poder generar acuerdos.

Carlos Gil-García

Universidad Autónoma de Hidalgo

Correo-e: cgilgarcia@yahoo.com

Recibida: 11 de septiembre de 2007. Aceptada: 17 de septiembre de 2007.

Carlos Gil-García. Es doctor en estudios latinoamericanos por la Universidad de París 3. Actualmente es profesor-investigador del Instituto de Ciencias Sociales y Humanidades de la Universidad Autónoma del Estado de Hidalgo, Área Académica de Ciencia Política y Administración Pública, y Coordinador del Programa de Maestría en Gobierno y Gestión Local de la misma institución. Su línea de investigación se centra en gobiernos locales y desarrollo sostenible. Entre sus publicaciones más recientes destacan: "La gobernanza urbana y el desarrollo sustentable en México: elementos para su análisis", ponencia presentada en el XI Congreso CLAD 2006, Ciudad de Guatemala, Guatemala. Las memorias fueron 
publicadas por el CLAD en CD, 15 pp.; "Acción pública urbano-ambiental. Conceptos para su análisis y un estudio de caso de las políticas urbano-ambientales: La ciudad de Aguascalientes, Ags. (1990-2002)", Investigación y Ciencia, 38, Universidad Autónoma de Aguascalientes, Ags., en prensa. 